\title{
Extranodal NK/T cell lymphoma mimicking Behçet's syndrome
}

Youyang Wang, Xiaoming Huang, Yang Jiao*

Department of General Internal Medicine, Peking Union Medical College Hospital, Chinese Academy of Medical Sciences and Peking Union Medical College, Beijing 100730, China

An 18-year-old man presented to our department with recurrent oral aphthous ulceration and a 1-month history of low-grade fever. On physical examination, there was painless ulceration of his glans penis (Figure 1A) and scrotum (Figure 1B) and erythematous plaques on his lower extremities. Behçet's syndrome was suspected and treated with low-dose glucocorticoids and thalidomide. However, his skin lesions failed to resolve and quickly progressed (Figure $1 \mathrm{C}$ and D). His erythrocyte sedimentation rate was normal. HIV, rapid plasma regain, and pathergy tests were all negative. Histopathological examination of the skin biopsy revealed a diagnosis of extranodal NK/T cell lymphoma (ENKTL), nasal type, for which he received chemotherapy.

ENKTL usually arises in midline facial structures and presents as localized disease. The clinical symptoms of the primary lesion are sometimes unremarkable, and penile metastases have only very rarely been reported. ${ }^{[1]}$ Behçet's syndrome is a multisystem vasculitis that can cause oral aphthous ulceration, genital ulceration, eye and skin lesions, and a positive pathergy test. ${ }^{[2,3]}$ Genital ulcers, often appearing on the scrotum in men, are highly specific for Behçet's syndrome; however, in our case, ENKTL with penile metastasis was an exceedingly rare mimic. The defined skin manifestations of Behçet's syndrome include erythema nodosum, pseudofolliculitis, acneiform nodules, papulopustular lesions, superficial thrombophlebitis, and palpable purpura ${ }^{[4]}$ but not the erythematous plaques seen in our case. The presence of atypical, progressive skin lesions should prompt further investigations to avoid diagnostic delay.
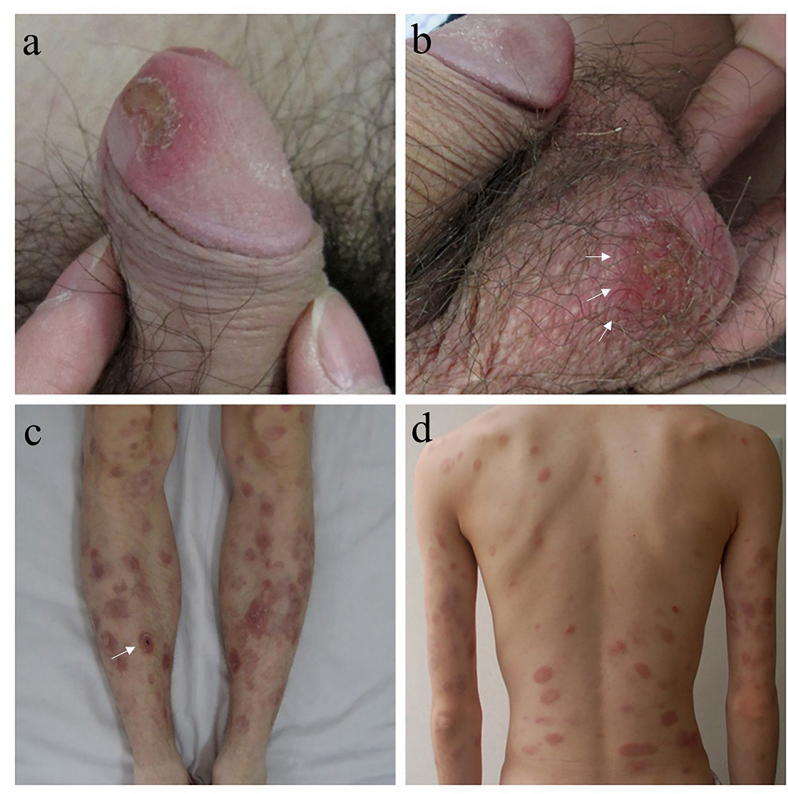

Figure 1. Ulceration of the patient's glans penis $(A)$, scrotum (arrow in $B$ ), and erythematous plaques or infiltrated papules (arrow in C) on the patient's lower extremities (C) and trunk (D).

\footnotetext{
Address for correspondence:

Yang Jiao, Department of General Internal Medicine, Peking Union Medical College Hospital, Chinese Academy of Medical Sciences and Peking Union Medical College, No. 1, Shuaifuyuan, Wangfujing Street, Beijing 100730, China; E-mail: peterpumch@163.com
} 
Ethical statement

This study was granted an exemption from review by the Institutional Review Board of Peking Union Medical College Hospital.

Conflict of interest

All authors declare that no conflict of interest exists.

Informed consent

Written informed consent was obtained from the patient for publication of this case report and the accompanying images.

Funding

China Medical Board Open Competition Program (20-384) and National Major Science and Technology Projects of China (Grant no. 2020YFC0841300) provided the financial support for editing and publication.

\section{References}

[1] Wang X, Gong Z, Li SX, et al. Extranodal Nasal-Type Natural Killer/T-cell Lymphoma with Penile Involvement: A Case Report and Review of the Literature. BMC Urol, 2017;17(1):77. doi: 10.1186/ s12894-017-0273-8.

[2] Nair JR, Moots RJ. Behcet's Disease. Clin Med (Lond), 2017;17(1):71-77. doi: 10.7861/clinmedicine.17-1-71.

[3] Greco A, De Virgilio A, Ralli M, et al. Behcet's Disease: New Insights into Pathophysiology, Clinical Features and Treatment
Options. Autoimmun Rev, 2018;17(6):567-575. doi: 10.1016/j.autrev.2017.12.006.

[4] Leccese P, Ozguler Y, Christensen R, et al. Management of Skin, Mucosa and Joint Involvement of Behcet's Syndrome: A Systematic Review for Update of the EULAR Recommendations for the Management of Behcet's Syndrome. Semin Arthritis Rheum, 2019;48: 752-762. doi: 10.1016/j.semarthrit.2018.05.008. 